\title{
Lysozyme improves gut performance and protects against enterotoxigenic Escherichia coli infection in neonatal piglets
}

\author{
Guangping Huang ${ }^{1}$, Xiangqing $\mathrm{Li}^{2,3}$, Dan $\mathrm{Lu}^{2,4}$, Shen $\mathrm{Liu}^{2,5}$, Xun Suo ${ }^{1}$, Qiuyan $\mathrm{Li}^{\mathrm{i}^{*}} \mathrm{C}$ and Ning $\mathrm{Li}^{2}$
}

\begin{abstract}
Diarrhea remains one of the leading causes of morbidity and mortality globally, with enterotoxigenic Escherichia coli (ETEC) constituting a major causative pathogen. The development of alternative treatments for diarrhea that do not involve chemotherapeutic drugs or result in antibiotic resistance is critical. Considering that lysozyme is a naturally occurring antimicrobial peptide, in a previous study we developed a transgenic pig line that expresses recombinant human lysozyme (hLZ) in its milk. In the present study, we examined the protective effects of the consumption of this milk against ETEC infection in neonatal piglets. We found that consuming hLZ milk facilitated faster recovery from infection and decreased mortality and morbidity following an ETEC oral inoculation or infection acquired by contactexposure. The protective effect of $h L Z$ was associated with the enrichment of intestinal bacteria that improve gut health, such as Lactobacillus, and the enhancement of the mucosal IgA response to the ETEC-induced diarrhea. Our study revealed potential protective mechanisms underlying the antimicrobial activity of human lysozyme, validating the use of lysozyme as an effective preventive measure for diarrhea.
\end{abstract}

\section{Introduction}

Enterotoxigenic Escherichia coli (ETEC) is one of the most devastating pathogens associated with diarrhea in animals and humans, annually accounting for 157000 deaths among children [1]. ETEC pathogenicity is dependent on colonizing factors (also known as adhesins) and enterotoxins [2]. ETEC that is associated with diarrhea in neonatal pigs expresses one or more fimbrial adhesins, including F4 (K88), F5 (K99), F6 (987P), F7 (F41) and F18, while ETEC in older pigs most commonly produces F4 (K88) or F18 fimbriae. Fimbrial adhesins bind to glycoprotein receptors on the enterocyte brush border, enabling $E$. coli colonization in the small intestine. The bacteria then secrete heat-labile enterotoxins and/or heat-stable enterotoxins that alter tight junction integrity and disrupt the paracellular passages of ions, solutes and water, leading to diarrhea [3, 4]. Persistent diarrhea results in dehydration and malnutrition, and

\footnotetext{
*Correspondence: liqiuyan-yuan@163.com

${ }^{2}$ State Key Laboratory of Agrobiotechnology \& College of Biological

Sciences, China Agricultural University, Beijing, China

Full list of author information is available at the end of the article
}

may increase the chance of subsequent infection by other pathogens. ETEC shed in the feces can survive for at least 5-6 months [5, 6], affording opportunities to contaminate other animals, plants, or water.

As widespread resistance is emerging for many antibiotics, the development of alternative diarrhea treatments that do not involve chemotherapeutic drugs or result in antibiotic resistance is crucial. Increasing studies have focused on the use of natural anti-bacterial proteins, such as lysozyme, lactoferrin and secretory IgA, which can be found in the tears, saliva, and milk of all mammals, and which provide non-specific antimicrobial and immune-stimulating activities [7-10]. As one of the main host defense factors, lysozyme directly kills bacteria by hydrolyzing the glycosidic $\beta-(1-4)$ linkage between $\mathrm{N}$-acetylmuramic acid and $\mathrm{N}$-acetylglucosamine of the peptidoglycan polymer in the cell wall [11-13]. In addition, lysozyme can also regulate immune function by directly or indirectly modulating the complement system, and can enhance the function and proliferation of polymorphonuclear neutrophils and phagocytes $[14,15]$. 
These effects suggest that lysozyme can be used to protect hosts against infectious diseases.

Although large volumes of cow and goat milk are available for human consumption, both are low in certain key health-promoting antimicrobial components such as lysozyme. Human milk contains a 1500- to 4000-fold more lysozyme $(400 \mathrm{mg} / \mathrm{L})$ than cow milk $(0.13 \mathrm{mg} / \mathrm{L})$ or goat milk $(0.25 \mathrm{mg} / \mathrm{L})$ [16]. The lysozyme concentration in pig milk is even lower, at less than $0.065 \mathrm{mg} / \mathrm{L} \mathrm{[17].}$ Advancements in gene transfer technologies have made it possible to breed transgenic cows and goats that express recombinant human lysozyme (hLZ) in their milk, incorporating this protective protein into a readily available product that can be used by both people and animals [10, $18-20]$.

We recently generated a line of transgenic pigs that express high levels of recombinant human lysozyme in their milk, with an average concentration of $1300.7 \pm 126.7 \mathrm{mg} / \mathrm{L}$ (range from 1140.5 to $1576.8 \mathrm{mg} / \mathrm{L}$ ) $[21,22]$. To test whether the milk confers protective effects, neonatal piglets were assigned to wild type sows or to sows that expressed human lysozyme in their milk. Half of the piglets were deliberately challenged with ETEC, and the other half was permitted to acquire the infection passively from their challenged littermates. Piglets were then monitored for diarrhea and other signs of pathology.

\section{Materials and methods}

\section{Bacterial strain and culture}

Enterotoxigenic Escherichia coli K88 (O149:K91, K88ac) was purchased from the China Veterinary Culture Collection Center (CVCC, Beijing, China). The virotype profile is O149:F4:LT:STb:Stx2e:eae [23]. Cultures were grown in Luria-Bertani (LB) medium containing $1 \%$ tryptone, $0.5 \%$ yeast extract and $1 \% \mathrm{NaCl}$, and incubated at $37{ }^{\circ} \mathrm{C}$ with shaking for $16 \mathrm{~h}$. Cultures were harvested by centrifugation at $3000 \times g$ for $10 \mathrm{~min}$, washed 3 times with sterile phosphate-buffered saline (PBS, $\mathrm{pH}=7.2$ ), re-suspended in sterile $\mathrm{PBS}$, and serially diluted to approximately $2 \times 10^{8} \mathrm{CFU} / \mathrm{mL}$ for oral challenge as described previously [24].

Micrococcus lysodeikticus and Staphylococcus aureus were purchased from the China General Microbiological Culture Collection Center (CGMCC, Beijing, China) and cultured in LB medium at 28 and $37{ }^{\circ} \mathrm{C}$, respectively, in shake flasks.

To measure the bactericidal effects of lysozyme, $2.5 \mathrm{~mL}$ of $E$. coli cell suspension with an optical density $\left(\mathrm{OD}_{600}\right)$ of approximately 0.7 was incubated with $100 \mu \mathrm{L}$ of defatted milk from hLZ-transgenic or wild type pigs. The inhibitory effect of lysozyme on the growth of Grampositive and Gram-negative bacteria was tested using $S$. aureus and E. coli, with $M$. lysodeikticus as a positive control. $\mathrm{OD}_{600}$ was recorded at $1,3,5$, and $7 \mathrm{~h}$ after incubation and compared with the value at $0 \mathrm{~h}$.

\section{Experimental design}

Five hLZ transgenic and 5 wild-type sows of the same parity were used in the experiment. All sows were confirmed seronegative for K88 antibodies using a commercial enzyme-linked immunosorbent assay (ELISA) kit, following the manufacturer's instructions (Shanghai Yaji Biological Technology Co., Ltd., Shanghai, China), as described previously [24]. Sixty wild-type female piglets with similar birth weights $(1.62 \pm 0.15 \mathrm{~kg})$ were randomly assigned to one of the ten sows (six piglets per sow) at 1 day of age. The environment was maintained at $30 \pm 2{ }^{\circ} \mathrm{C}$ and all piglets were allowed to nurse. During the 17-day lactation period, sow milk was the only source of nutrition for the piglets. All piglets had free access to water.

For each sow, three of the six piglets were orally challenged at 3 days of age with a dose of $5 \mathrm{~mL}$ PBS containing approximately $1 \times 10^{9} \mathrm{CFU}$ of ETEC O149: $\mathrm{K} 88$, delivered via a stomach tube connected to a $5 \mathrm{~mL}$ syringe. These three piglets are hereafter described as "challenged". The dose of ETEC used in this study causes diarrhea within $6 \mathrm{~h}$ in young piglets (data not shown). After receiving the ETEC, the three piglets were returned to their pen in order to contact-expose their three littermates, referred to throughout the manuscript as cohabited piglets. The beginning of the experiment is defined at this point (time $=0$ ). In summary, the piglets were assigned to four experimental groups of 15 piglets each, defined by two milk sources (hLZ transgenic milk [hLZ] vs. wild-type milk feeding [WT]), and two infection states (ETEC challenged vs. cohabited).

After 7 days, the challenged piglets were euthanized, and the three cohabited piglets remained with their assigned sow. Piglets were weighed on days $0,3,5,7$, and 14.

\section{Clinical scoring}

Disease signs were scored as described in a previous study scored as described in a previous study [25]. Piglets were monitored daily by a pathologist blinded to the treatments. A disease score of 0 indicated no signs of diarrhea, lethargy or dehydration; 1, feces softer than normal, but with overall demeanor unaffected; 2, feces liquid with little or no lethargy or dehydration; 3 , appearance of prolonged diarrhea, with obvious dehydration and/or lethargy; and 4, diarrhea with severe, life-threatening dehydration and lethargy. Piglets were considered dehydrated if a pinch of skin at the base of the neck did not return to its original shape. Piglets were identified as 
lethargic if they were motionless in the pen or required stimulation to move. Piglets were considered to have diarrhea when the disease score was at a level of 2 or greater, and piglets with a disease score of 4 for at least 2 consecutive days were recorded as deaths and humanely euthanized with an intraperitoneal injection of pentobarbitone to minimize suffering [25], as they were unlikely to recover. Rectal temperature was registered daily from the challenge day onwards, and measurements were taken prior to any other manipulation. The incidence of diarrhea (\%) was calculated as a percentage of the number of newly affected piglets during the experimental period divided by the total number of piglets in each group [25].

\section{Detection of fecal ETEC K88 from challenged piglets by polymerase chain reaction (PCR)}

Fecal samples from challenged piglets were examined for ETEC K88 $6 \mathrm{~h}$ after the challenge. Bacterial DNA was extracted using a QIAmp DNA stool kit (Qiagen, Valencia, CA, USA), following the manufacturer's instructions. Adhesin gene F4 was detected by PCR using forward primer 5'-GCTGCATCTGCTGCATCTGGTATGG-3' and reverse primer $5^{\prime}$-CCACTGAGTGCTGGTAGTTACAGCC-3', as described previously [26].

\section{Histological examination of the intestinal morphology}

All cohabitated piglets were euthanized on day 14 of the experiment. The small intestine was divided into three segments: duodenum, to about $5-8 \mathrm{~cm}$ from the pylorus; jejunum, the middle portion; and ileum, the distal section approximately $5 \mathrm{~cm}$ in length proximal to the ileocecal junction. After being gently rinsed with ice-cold PBS $(\mathrm{pH}=7.2)$, the segments were immediately fixed in $4 \%$ paraformaldehyde, embedded in paraffin, sliced into $4 \mu \mathrm{m}$ thick sections, and stained with hematoxylin and eosin. Villus length and crypt depth were measured using Image-Pro Plus software (Image-Pro Plus 6.0; Media Cybernetics, Silver Spring, MD, USA). Ten well-oriented villi were selected and measured in triplicate for each piglet.

\section{Analysis of the distribution and expression of tight junction proteins by real-time PCR}

The tight junction (TJ) is formed by a protein complex and is widely used as a marker of intestinal integrity [27]. Mucosal tissues from the duodenum, jejunum and ileum were scraped with a glass slide, immediately placed in liquid nitrogen, and stored at $-80{ }^{\circ} \mathrm{C}$. Real-time PCR analysis for TJ mRNA expression was performed using a SYBR Premix Ex Taq II qPCR kit (TaKaRa Biotechnology, Dalian, China) on a LightCycler480 thermocycler (Roche, Mannheim, Germany). Gene-specific primers for the amplicons of zonula occludens (ZO)-1, occludin, and $\beta$-actin are listed in Table 1 . Relative transcript levels were quantified by the $2^{-\Delta \Delta \mathrm{CT}}$ method as described previously [28].

\section{Analysis of gut microbiota by $16 \mathrm{~S}$ rRNA sequencing}

Fresh fecal samples were obtained on days $0,3,5,7$, and 14 from the cohabitated piglets in each feeding group. The collected material was mixed well to produce a single homogeneous sample, and then divided into 3 equal parts. All fecal samples were stored at $-80{ }^{\circ} \mathrm{C}$ prior to bacterial DNA extraction. The V3 hypervariable region of the 16S rRNA gene was amplified by PCR using a forward primer ( $5^{\prime}$-GATCCTACGGGAGGCAGCA-3 $\left.{ }^{\prime}\right)$ and reverse primer (5'-GCTTACCGCGGCTGCTGGC-3') [29]. Sequencing libraries were generated using the NEBNext $^{\circledR}$ Ultra $^{\mathrm{TM}}$ DNA Library Prep Kit (NEB, USA), following the manufacturer's recommendations. The library was sequenced on an Illumina MiSeq platform [30].

To analyze gut microbial diversity, operational taxonomic units (OTUs) were clustered with a 97\% similarity cutoff using UPARSE (version 7.1). The taxonomy of each 16S rRNA gene sequence was assigned using the Ribosomal Database Project Classifier against the Silva (SSU128) 16S rRNA database with a confidence threshold of $70 \%$ [31]. Metastats was used to confirm differences in taxonomic abundance between the two groups.

\section{Quantitation of IgA and pro-inflammatory cytokines by ELISA}

Fecal extracts collected on days 7 and 14 were concentrated fourfold by freeze-drying and resuspending with sterile water. Secretory IgA (sIgA) antibody to E. coli $\mathrm{K} 88 \mathrm{ac}$ was quantified using indirect ELISA assay kits from Shanghai Yaji Biological Technology Co., Ltd., Shanghai, China. Serum was separated from clotted blood on days 0,7 , and 14 and preserved at $-20{ }^{\circ} \mathrm{C}$ until use. IL- 6 and TNF- $\alpha$ were quantitated using commercially available ELISA kits (Shanghai Yaji Biological Technology Co., Ltd., Shanghai, China). All ELISA procedures were performed as recommended by the manufacturer's instructions. Each sample was assayed in triplicate.

\section{Table 1 Primers used for real-time PCR analysis}

\begin{tabular}{lll}
\hline Gene & Primers sequences & Size in base pairs \\
\hline ZO-1 & F: 5'-TGAGTTTGATAGTGGCGTTG-3' & 298 \\
& R: 5'-TGGGAGGATGCTGTTGTC-3' & \\
Occludin & F: 5'-CTAGTCGGGTTCGTTTCC-3' & 167 \\
& R: 5'-GACTGATTGCCTAGAGTGT-3' \\
B-actin & F: 5'-TGCGGGACATCAAGGAGAAGC-3' & 273 \\
& R: 5'-ACAGCACCGTGTTGGCGTAGAG-3' & \\
\hline
\end{tabular}




\section{White blood cell (WBC) counts}

White blood cell subpopulations (including lymphocytes, monocytes, and neutrophils) were quantified with a Hemavet hematology analyzer (Drew Scientific, Dallas, Texas, USA).

\section{Statistical analysis}

All experimental data were analyzed using SPSS software (V. 19.0; SPSS Inc., Chicago, IL, USA). Data were expressed as mean \pm SEM. Statistical significance was determined using two-tailed Student's unpaired $t$ test. Differences were considered significant at $P$ values less than 0.05 .

\section{Results}

Reduced severity and duration of diarrhea in ETEC K88 challenged and cohabitated piglets fed with hLZ milk Challenged infection

All piglets in WT milk feeding group and hLZ milk-feeding group exhibited diarrhea (score $\geq 2$ ) within $6 \mathrm{~h}$ after the challenge with ETEC K88 (Figure 1A). PCR analysis indicated that all challenged pigs excreted ETEC in their feces (data not shown). Compared to the hLZ-fed group, piglets in the WT-fed group showed more severe and extensive diarrhea, especially by days 3 and $5(P<0.05$, Figure 1A). Twelve out of 15 piglets $(80 \%)$ belonging to the WT-fed group displayed high fever $\left(>40^{\circ} \mathrm{C}, P<0.05\right)$ and growth retardation (Figures $1 \mathrm{~B}$ and $\mathrm{C}$ ). Six piglets $(40 \%)$ displayed severe diarrhea (score $=4)$ with lifethreatening dehydration and apparent lethargy, and were considered as deaths and euthanized on days 3 and 5 (Figure 1D). However, most of the piglets $(73.4 \%, 11 / 15)$ that consumed hLZ transgenic milk suffered from mild diarrhea (score $=2$ ), and their symptoms abated 3 days later. Only 2 piglets $(13.3 \%$, score $=4)$ failed to recover from the diarrhea and were euthanized on days 3 and 5 (score $=4$, Figure 1D). Notably, pigs treated with the hLZ milk appeared to resume growth on day 5 (Figure 1C). All challenged piglets were euthanized on day 7 .

\section{Cohabitation infection}

The cohabitated (i.e., unchallenged) piglets were contactexposed to the challenged piglets from the onset of the experiment. Within 7 days, all cohabited piglets in both feeding groups showed subnormal body weight gain and increased body temperature (Figures $1 \mathrm{E}-\mathrm{G}$ ). Diarrhea (score $\geq 2$ ) occurred in 12 out of $15(80 \%)$ cohabitating piglets fed with WT milk, and it persisted for at least 2 days longer $(P<0.05$, Figure $1 \mathrm{E})$, along with a higher body temperature $(P<0.05$, Figure $1 \mathrm{~F})$ and poor growth (Figure 1G), compared with the hLZ-fed group. Diarrheal symptoms became aggravated in 2 piglets, resulting in death on day $5(13.3 \%$; score $=4$, Figure $1 \mathrm{H})$. In contrast, the hLZ-fed group displayed transient diarrhea and most diarrheal piglets were apparently healthy after exposure to ETEC-contaminated feces. Only one piglet (6.7\%) developed severe diarrhea (score $=4$, Figure $1 \mathrm{H}$ ).

Overall, piglets in the hLZ-fed group experienced diarrhea for a shorter time, with lower clinical scores and incidence than the piglets in the WT-fed group, suggesting that the consumption of human lysozyme improved the outcome.

\section{Lysozyme protects against ETEC-induced intestinal mucosal damage}

The morphology of three intestinal sections from the cohabitated piglets was examined at the end of the experiment (day 14; Figure 2A). Milk source effects were assessed using measurements of villus length, crypt depth, and the ratio (villus length/crypt depth) of these values (Figures $2 \mathrm{~B}$ and $\mathrm{C}$ ). In the jejunum and ileum, the ratio was significantly higher in hLZ fed than in WT fed piglets. However, the ratio in the duodenum was statistically indistinguishable between the two feeding groups, although it is slightly higher in hLZ fed animals. We conclude that the WT milk-fed piglets display obvious pathological damage, including microvillus loss and eosinophil infiltration (Figure 2A), in comparison with the hLZ fed group.

To further evaluate the integrity of the intestinal apical membrane, the expression of $\mathrm{ZO}-1$ and occludin were measured at the end of the experiment using quantitative real-time PCR. Compared to hLZ milk-fed piglets, ZO-1 and occludin were significantly depleted in the WT-fed group, especially in the jejunum and ileum (Figure 3), indicating that $\mathrm{hLZ}$ protects piglets from ETEC K88induced severe intestinal mucosal injury.

Together, these results suggest that hLZ milk positively affects intestinal morphology without compromising the integrity of the $\mathrm{TJ}$ in the apical membrane.

\section{Protection against ETEC infection does not depend on the lytic function of lysozyme}

Cell wall destruction by lysozyme in Gram-negative bacteria is less severe than Gram-positive bacteria due to the protective effects of outer membrane lipopolysaccharides. Although the concentration of lysozyme in the transgenic milk is more than $1000 \mathrm{mg} / \mathrm{L}$, the inhibitory effects on the growth of K88 in vitro were negligible (Figure 4). In contrast, the growth of the Gram-positive species M. lysodeikticus $(P<0.05)$ and $S$. aureus $(P<0.05)$ was significantly inhibited. $M$. lysodeikticus was considerably more sensitive to lysozyme since the cell walls are composed of a peptidoglycan polymer. 


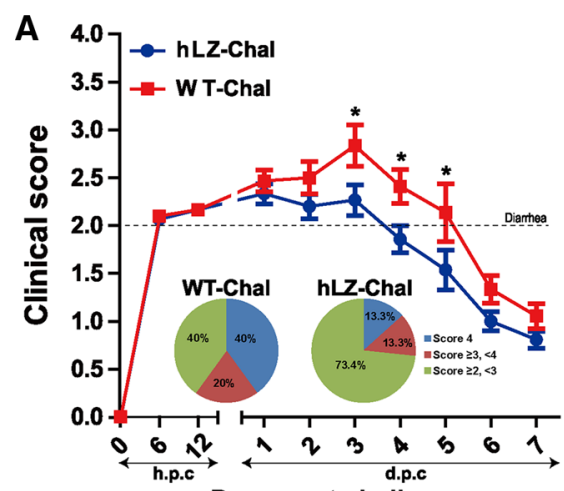

Days post challenge

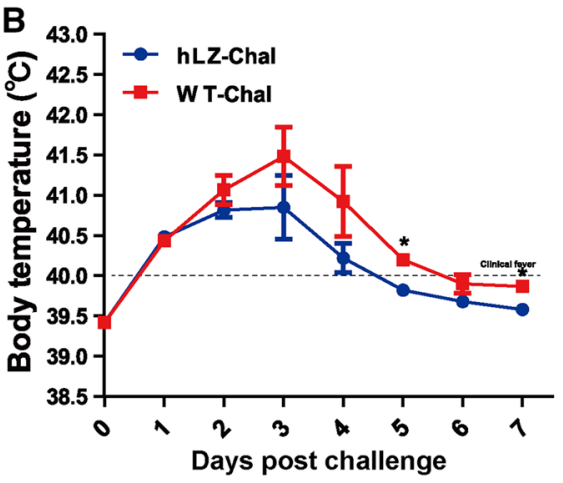

C

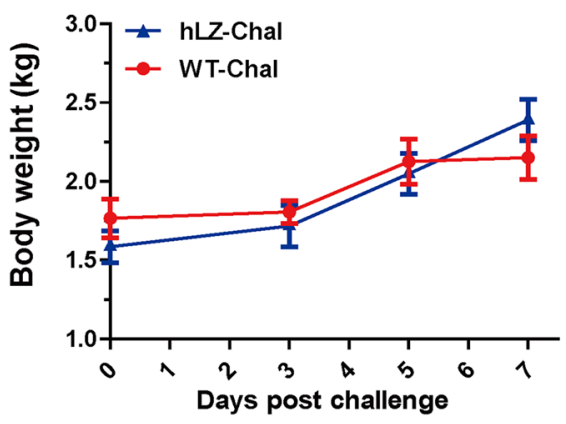

D

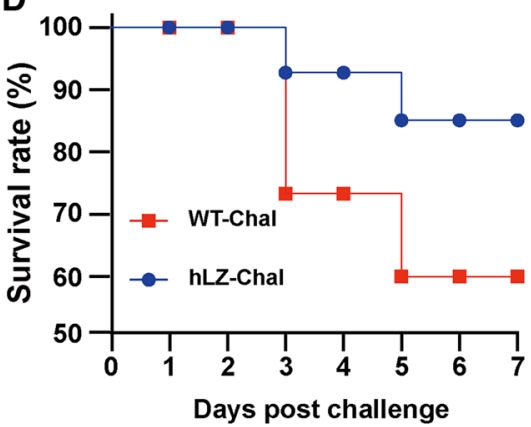

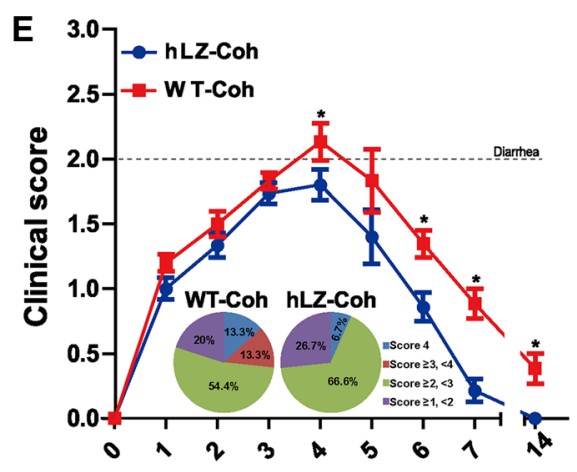

Days post cohabitation

F
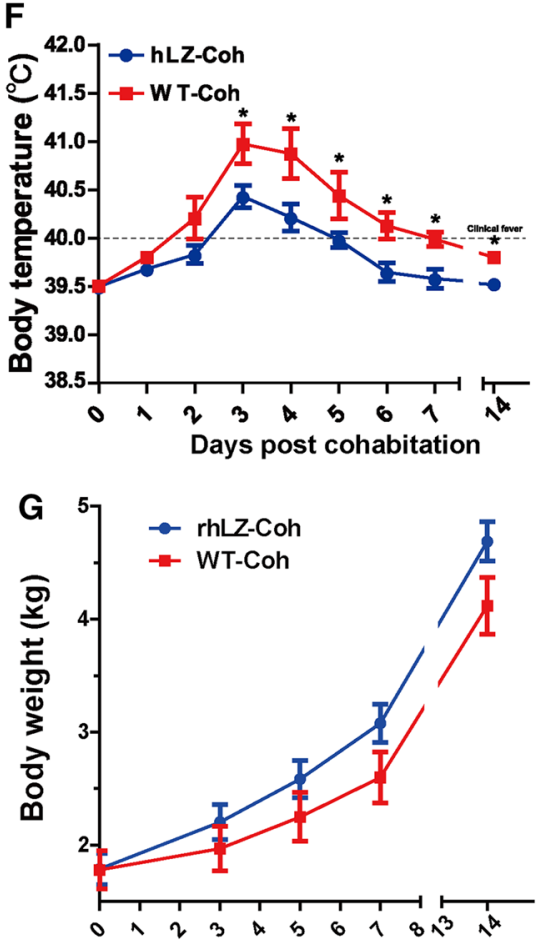

Days post cohabitation

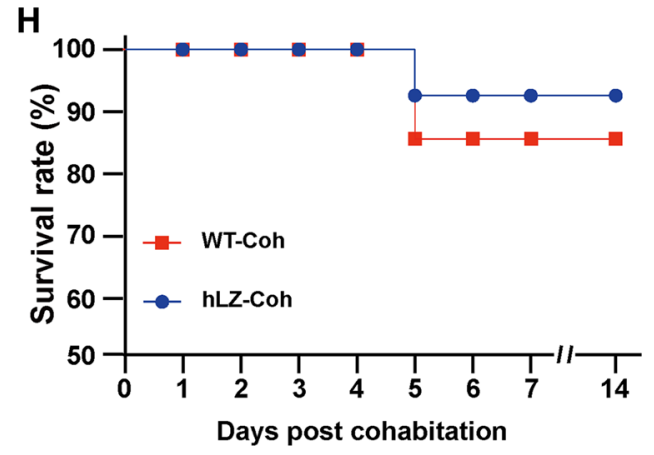

Figure 1 Piglets fed with hLZ milk were protected from ETEC. The left column A-D represents data collected from ETEC challenged piglets, and the right column $\mathbf{E}-\mathbf{H}$ represents cohabited piglets that passively acquired ETEC from challenged littermates. Time zero is defined as the point at which piglets were challenged and penned with the unchallenged (cohabited) piglets. Clinical scores for challenged (A) or cohabitated (E) piglets. Rectal temperature was monitored daily $(\mathbf{B}, \mathbf{F})$. The body weight gains were measured at the indicated times $(\mathbf{C}, \mathbf{G})$. Survival rates $(\mathbf{D}, \mathbf{H})$. Note that all challenged piglets were euthanized on day 7. Asterisk indicates $P<0.05$. hLZ: hLZ milk-fed piglets, WT: control milk-fed piglets, Chal: challenged, Coh: cohabited. 

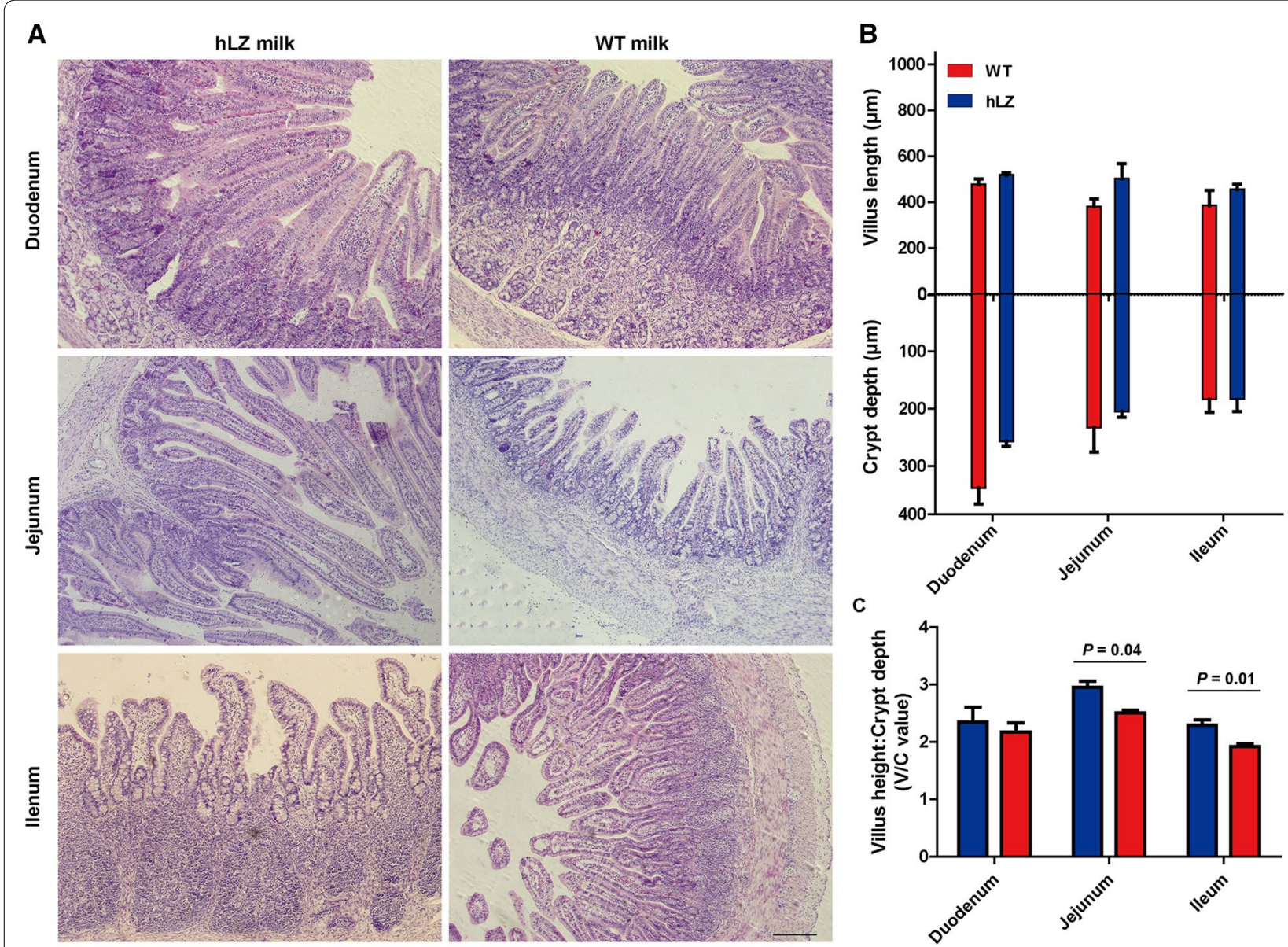

Figure $\mathbf{2}$ hLZ alleviates ETEC-induced intestinal damage. A Representative histological sections of the intestinal segments from cohabited piglets fed hLZ milk or wild type milk. Tissues were collected on day 14. Scale-bar $=200 \mu \mathrm{m}$. Villus length and crypt depth (B) and the ratios of villus length to crypt depth $(\mathbf{C})$ at the duodenum, jejunum and ileum from the piglets fed with hLZ or WT milk are shown as mean $\pm \operatorname{SEM}(n=10)$.
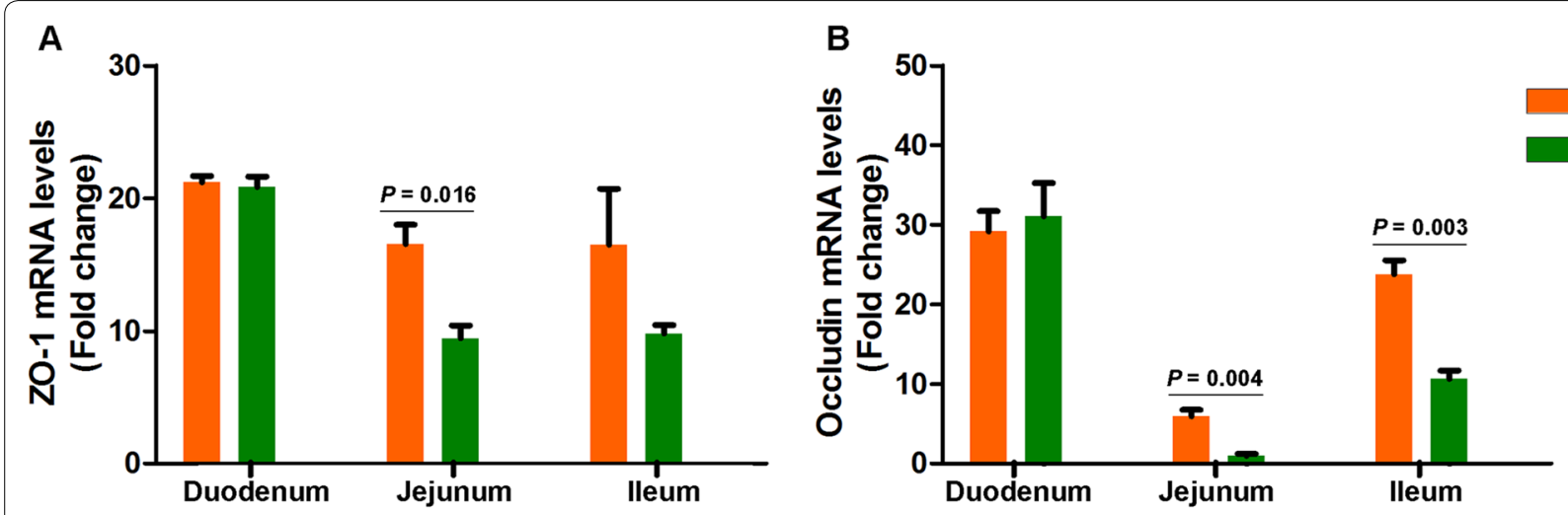

Figure 3 Tight junction proteins ZO-1 (A) and Occludin (B) mRNA levels in the intestinal mucosa (including duodenum, jejunum, and ileum) of cohabited piglets determined by real-time PCR. Data are shown as mean values \pm SEM. $P<0.05$ was considered statistically significant. hLZ: hLZ milk-fed piglets, WT: control milk-fed piglets. 


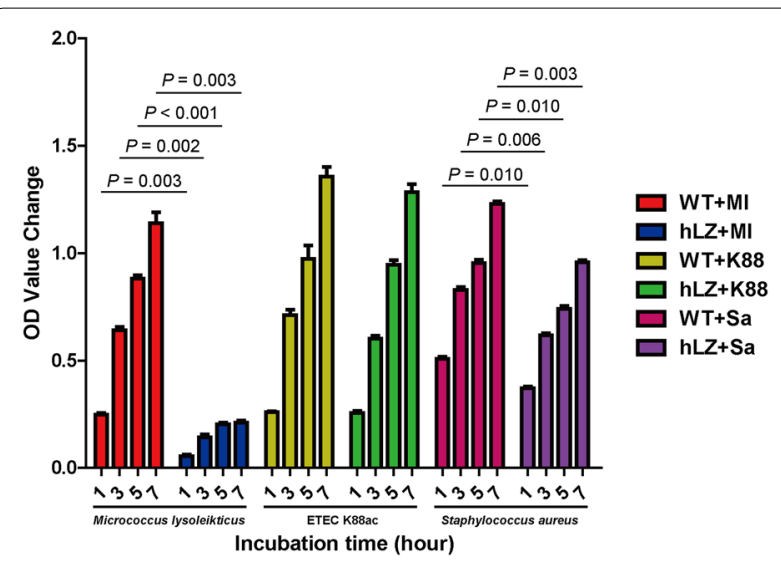

Figure $4 \mathrm{hLZ}$ inhibits the growth of Gram-positive bacteria (Micrococcus lysodeikticus and Staphylococcus aureus) with less influence on Gram-negative bacteria (Escherichia coli K88). Bacteria were cultured in Luria-Bertani medium for $8 \mathrm{~h}$, diluted to approximately $2000 \mathrm{CFU} / \mathrm{mL}$, and incubated with $100 \mu \mathrm{L}$ of defatted milk from transgenic pigs. Controls were incubated with the same volume of defatted WT milk. $\mathrm{OD}_{600}$ was recorded after 1, 3, 5, and $7 \mathrm{~h}$ and then compared with the value at $0 \mathrm{~h}$. WT + Ml: Micrococcus lysodeikticus treated with WT milk; hLZ + MI: Micrococcus lysodeikticus treated with hLZ transgenic milk; WT + K88: Escherichia coli K88 incubated with WT milk; hLZ + K88: Escherichia coli K88 incubated with hLZ milk; WT + Sa: Staphylococcus aureus incubated with WT milk; hLZ + Sa: Staphylococcus aureus incubated with hLZ milk. $P<0.05$ was considered statistically significant.

\section{Significant enrichment of probiotics in the gut is associated with lysozyme consumption}

To examine the effects of hLZ milk on intestinal flora, changes in the intestinal microbiome were monitored using $16 \mathrm{~S}$ rRNA gene sequencing. Fecal microbial profile showed that all piglets started with similar fecal microbial populations, but the profiles changed significantly after 5 days of hLZ milk consumption (Figure 5A). Animals receiving hLZ milk had an underrepresentation of Bacteroidetes were underrepresented and Firmicutes were overrepresented in the animals receiving hLZ milk (Figure 5A). Within the Firmicutes, the genus Lactobacillus in hLZ-fed pigs was present in a significantly greater proportion and was apparently higher than in WT-fed piglets on both days 7 and $14(P<0.05$, Figures $5 \mathrm{~B}$ and C). Conversely, three genera (Clostridium, Streptococcus, and Escherichia) that are associated with disease apparently increased over time in WT-fed pigs (Figure 5B). The results demonstrated that hLZ milk modulated intestinal microbiota by increasing the ratio of beneficial bacteria and decreasing the numbers of disease-causing microbe.

\section{An enhanced mucosal immune response plays a crucial role in the protection against ETEC infection}

sIgA is an indicator of mucosal immunology. Fecal sIgA in the WT- and hLZ-fed groups was assayed on days 7 and 14. We found that the sIgA levels in hLZ milk-fed piglets were slightly higher on day 7 and increased significantly on day $14(P=0.037)$ relative to WT-fed control (Figure 6), indicating that the mucosal immune response was enhanced following the consumption of hLZ milk.

\section{Decreased inflammatory response to ETEC K88 infection in hLZ-fed pigs}

The secretion of pro-inflammatory cytokines IL-6 and TNF- $\alpha$ in cohabiting piglets increased after exposure to ETEC-contaminated feces. However, piglets fed hLZmilk showed significantly lower serum levels of both IL-6 $(P<0.05)$ and TNF- $\alpha(P<0.05) 7$ and 14 days after exposure to ETEC, indicating a limited inflammatory response to ETEC infection (Figures $7 \mathrm{~A}$ and $\mathrm{B}$ ).

WBC count analysis showed that hLZ-milk fed piglets tended to have significantly more neutrophils $(P<0.05)$ and fewer lymphocytes and monocytes than those of WT-fed piglets from day 3 after exposure to ETEC (Figures 7C-E).

\section{Discussion}

We examined the ability of lysozyme, a natural antimicrobial protein, to protect neonatal piglets against ETEC K88 infection. We found that the consumption of hLZ milk helped ETEC-challenged piglets to recover from infection faster with lower mortality, and helped cohabitated piglets to decrease the morbidity with less intestinal damage, and enhanced IgA production and Lactobacillus enrichment. These findings demonstrate that the introduction of human lysozyme played a beneficial role in gut health and improved the ability of the piglets to resist bacterial infections.

Although lysozyme is well known for its robust antimicrobial activity against Gram-positive bacteria, and to a much lesser degree against Gram-negative bacteria, its bactericidal effect on ETEC K88 in vitro is modest due to the presence of lipopolysaccharides on the outer membrane of E. coli K88 (Figure 4) [31-33]. The protective effects observed in vivo against ETEC K88 infection could therefore be due to the regulatory function of lysozyme in the intestinal environment.

Interestingly, nonpathogenic bacteria often encode Ivy, PgdA, Oat, and Pat $O$-acetyltransferases, which are involved in regulating lysozyme resistance [34]. Consistent with a report that found that the consumption of lysozyme-rich milk contributes to the development of intestinal bacteria associated with gut health [19], we found an increase in the abundance of Lactobacillus in hLZ-fed piglets. Lactobacillus has been shown to improve the intestinal environment and activate intestinal mucosal immunity in many species, resulting in enhanced IgA production [35, 36], which is required 


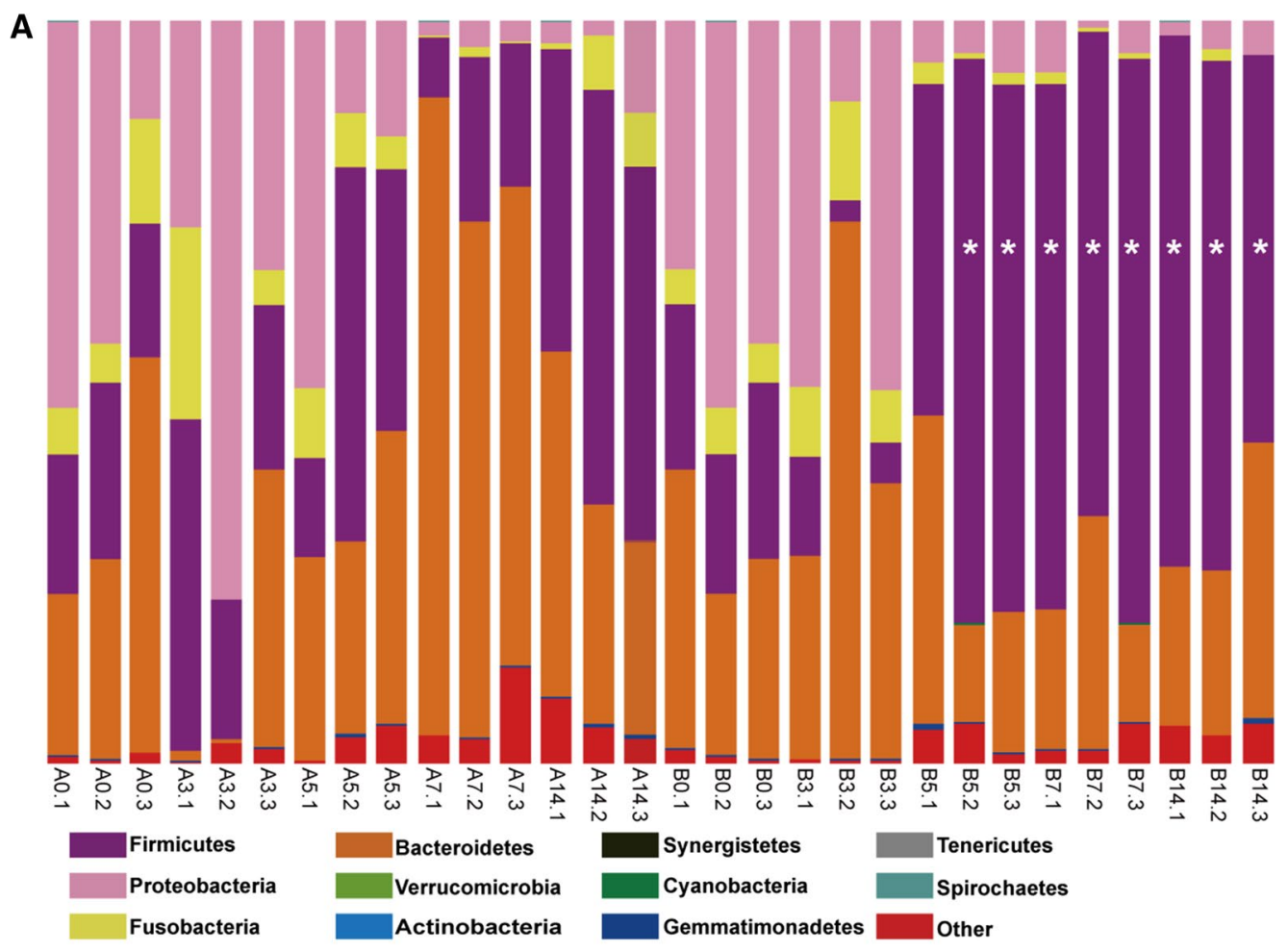

B

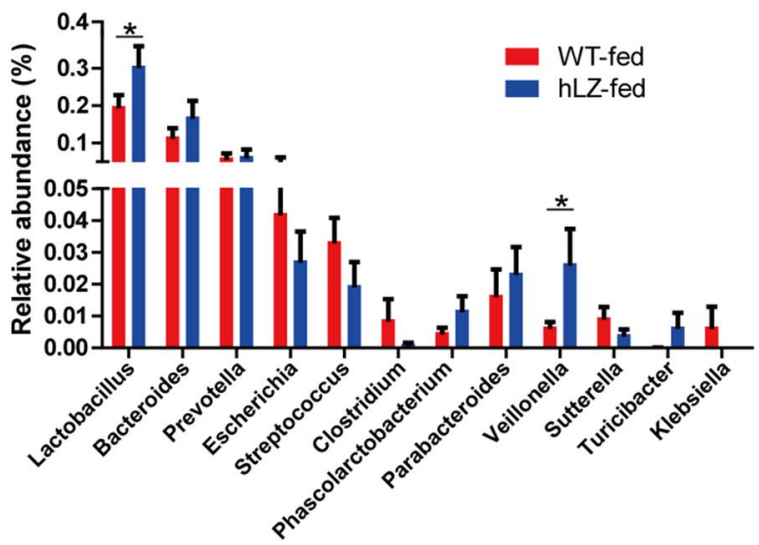

C

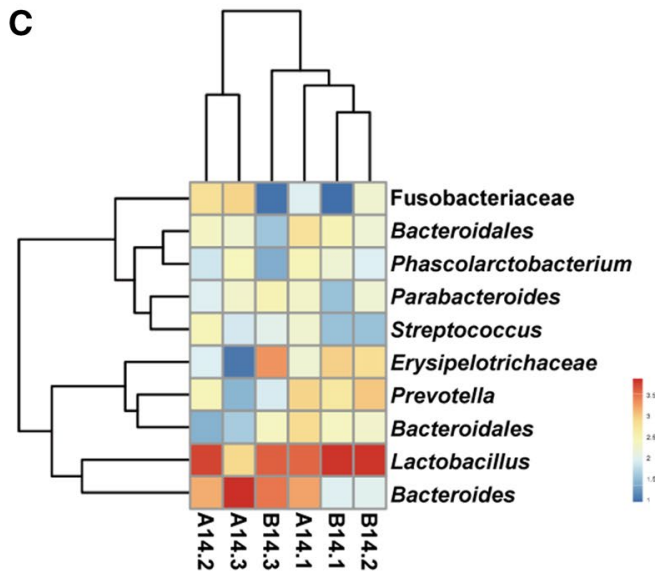

Figure 5 Consumption of hLZ milk increases the abundance of probiotics (Lactobacillus) in the intestine of cohabited piglets. A Relative phylum abundance of gut microbiota from WT and hLZ milk-fed piglets on days $0,3,5,7$, and 14 . B Relative abundance of bacterial genera in the guts of WT and hLZ milk-fed piglets at day 14. C Taxonomic classifications at the genus level for the fecal samples collected at day 14. Only the 10 most enriched classes are shown. A The fecal samples collected from WT milk-fed piglets at days 0 (A0), 3 (A3), 5 (A5) 7 (A7) and 14 (A14); B the fecal samples collected from hLZ milk-fed piglets at days 0 (B0), 3 (B3), 5 (B5) 7 (B7) and 14 (B14). The samples from each day were divided into three equal parts and measured as replicates. Asterisk indicates $P<0.05$.

for the prevention of fimbriae-mediated colonization and the maintenance of intestinal barrier function. In contrast, ETEC infection in the WT-feeding group increased the levels of Clostridium, Streptococcus, and Escherichia, typically characterized as opportunistic pathogenic bacteria [37]. Lysozyme is also important for limiting systemic inflammation, resulting in decreased inflammatory-driven pathology [34, 38, 39], which is consistent with the lower production of pro-inflammatory cytokines in the hLZ-fed pigs in our study.

ETEC is a significant cause of diarrhea either in neonatal or early-weaned piglets, resulting in severe economic losses in the swine industry [40]. Although several vaccines have been successfully developed for weaned pigs, 


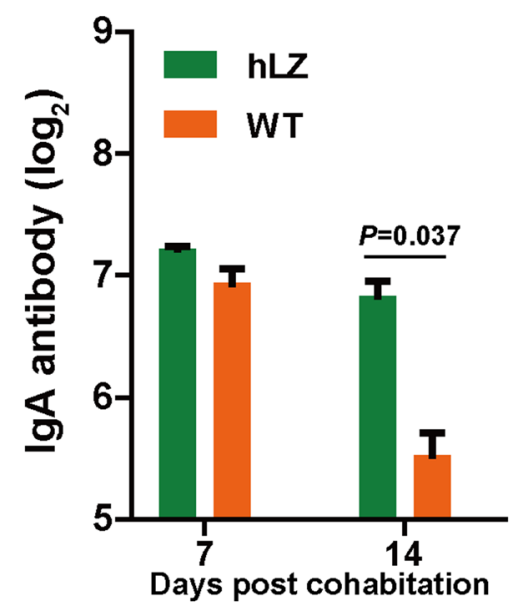

Figure 6 hLZ enhances mucosal IgA immune response to ETEC K88 infection. $h L Z$ : hLZ milk-fed piglets, WT: WT milk-fed piglets.

diarrhea always occurs early after weaning, often within 3-10 days [41]. It would be ideal to vaccinate during the sucking period. However, apart from the ability to resist gastrointestinal degradation, another challenge for ETEC vaccines is to overcome neutralization by maternal antibodies and/or other milk factors [41].

The use of antibiotics at sub-therapeutic levels throughout the swine industry improves performance and overall health. However, increasing concerns regarding antibiotic resistance have made their use problematic [42], and research into alternatives is therefore essential. Lysozyme, as a natural antimicrobial treatment, has been extensively used as a feed additive to increase growth and feed efficiency [43]. As shown in our study, the consumption of lysozyme by neonatal pigs enriches intestinal commensal bacteria and enhances the mucosal IgA response that reinforces the mucus barrier to prevent the penetration of ETEC K88 into epithelial cell surfaces. Furthermore, the high level of neutrophils contributes to the clearance of ETEC, leading to decreased pro-inflammatory cytokine production and limited E. coli-induced inflammation. Our findings indicate that lysozyme may be a viable alternative to traditional sub-therapeutic antibiotic use in swine production. To date, multiple lines of transgenic dairy animals that produce lysozyme have been developed to improve animal welfare and growth performance [10, 18, 20-22, 44, 45]. Recent advances in transgenic technologies have removed many of the technical barriers to the predictable and efficient genetic engineering of agricultural species, although there are many political and regulatory hurdles to overcome before genetic engineering can be used in agriculture $[46,47]$.

In conclusion, the data in this study demonstrate that human lysozyme is beneficial for the gut performance of neonatal piglets and improves resistance to bacterial infections, providing an effective preventive measure for diarrhea.

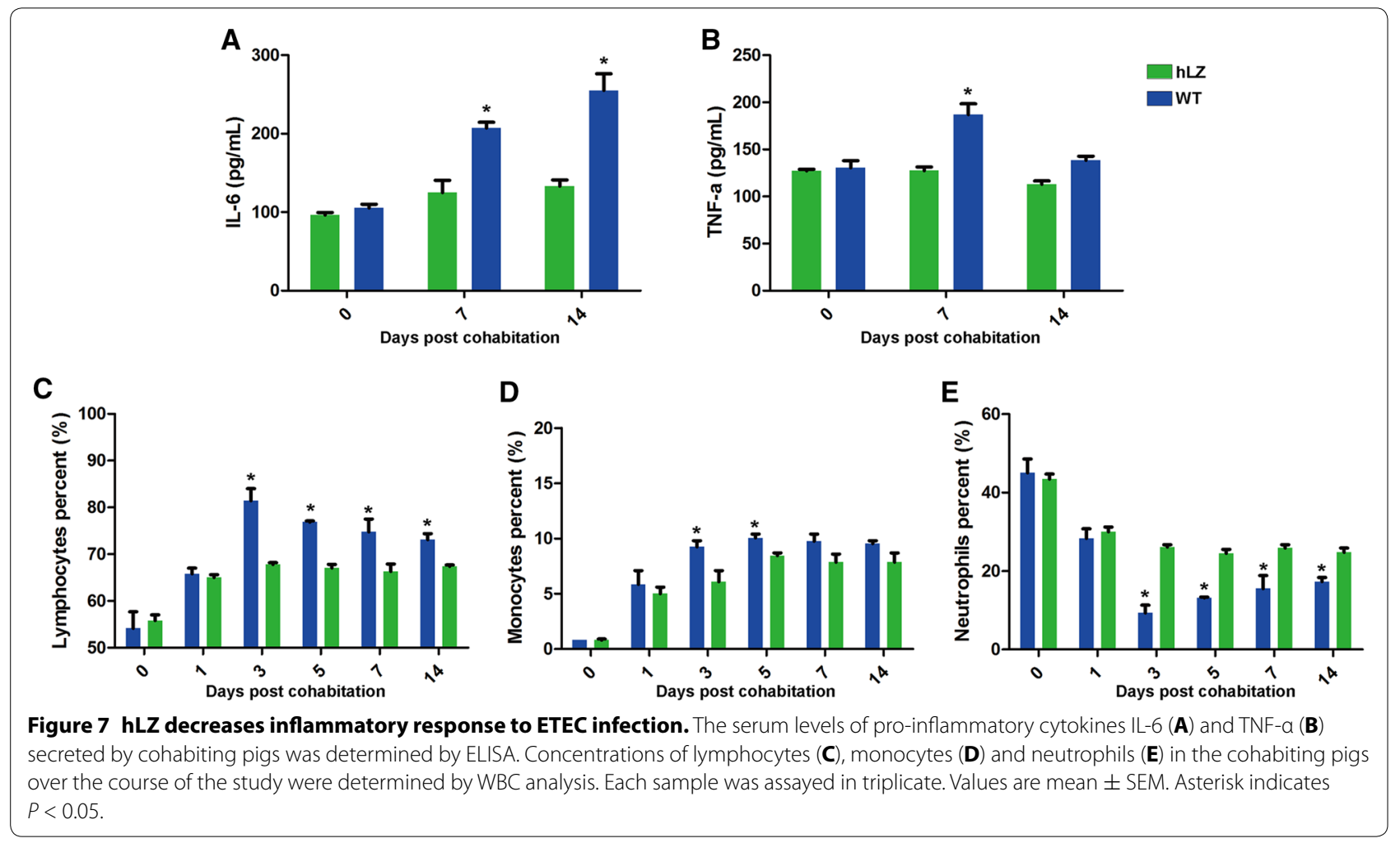




\section{Authors' contributions}

The study was conceived and experiments designed by QL, NL and GH. The experiments were performed by $\mathrm{GH}, \mathrm{XL}$ and $\mathrm{DL}$. Data were analyzed and interpreted by QL, XS, DL, SL and GH. QL, XS and NL contributed to the reagents, materials, or analysis tools. While GH drafted the paper, and all other authors revised the manuscript for important intellectual content. All authors read and approved the final manuscript.

\section{Acknowledgements}

We wish to thank Zaihu Zhang, Jie Suo, Yijing Fu, Jie Luo, Haiyang Hao, Jianhu Bai, Yunzhou Wang, Xiaoli Tang, and Zhiyuan Li for their excellent technical assistance. We thank LetPub for providing linguistic assistance during the preparation of this manuscript.

\section{Competing interests}

The authors declare that they have no competing interests.

\section{Ethics approval and consent to participate}

All experiments performed in this study were in strict accordance with the Guide for the Care and Use of Laboratory Animals of the Ministry of Science and Technology of China, and were approved by the Institutional Animal Care and Use Committee of China Agricultural University (ID: 1114120800083). All efforts were made to minimize animal suffering.

\section{Funding}

This study was supported by the National Transgenic Breeding Program of China (No. 2016ZX08006003, 2014ZX08006006 and 2014ZX0800603B).

\section{Author details}

1 State Key Laboratory of Agrobiotechnology \& College of Veterinary Medicine, China Agricultural University, Beijing, China. ${ }^{2}$ State Key Laboratory of Agrobiotechnology \& College of Biological Sciences, China Agricultural University, Beijing, China. ${ }^{3}$ Shenzhen Sunsmile Biotechnology Co., Ltd, Shenzhen, Guangdong, China. ${ }^{4}$ Shanghai Institute of Medical Genetics, Shanghai Children's Hospital, Shanghai, China. ${ }^{5}$ School of Life Science and Engineering, Foshan University, Foshan, Guangdong, China.

\section{Publisher's Note}

Springer Nature remains neutral with regard to jurisdictional claims in published maps and institutional affiliations.

Received: 23 October 2017 Accepted: 25 January 2018

Published online: 20 February 2018

\section{References}

1. Kotloff KL, Nataro JP, Blackwelder WC, Nasrin D, Farag TH, Panchalingam S, Wu Y, Sow SO, Sur D, Breiman RF, Faruque AS, Zaidi AK, Saha D, Alonso PL, Tamboura B, Sanogo D, Onwuchekwa U, Manna B, Ramamurthy T, Kanungo S, Ochieng JB, Omore R, Oundo JO, Hossain A, Das SK, Ahmed S, Qureshi S, Quadri F, Adegbola RA, Antonio M et al (2013) Burden and aetiology of diarrhoeal disease in infants and young children in developing countries (the Global Enteric Multicenter Study, GEMS): a prospective, case-control study. Lancet 382:209-222

2. Kaper JB, Nataro JP, Mobley HL (2004) Pathogenic Escherichia coli. Nat Rev Microbiol 2:123-140

3. Wilson RA, Francis DH (1986) Fimbriae and enterotoxins associated with Escherichia coli serogroups isolated from pigs with colibacillosis. Am J Vet Res 47:213-217

4. Ruan X, Zhang W (2013) Oral immunization of a live attenuated Escherichia coli strain expressing a holotoxin-structured adhesin-toxoid fusion (1FaeG-FedF-LTA2:5LT(B)) protected young pigs against enterotoxigenic E. coli (ETEC) infection. Vaccine 31:1458-1463

5. Walker CLF, Rudan I, Liu L, Nair H, Theodoratou E, Bhutta ZA, O'Brien KL, Campbell H, Black RE (2013) Global burden of childhood pneumonia and diarrhoea. Lancet 381:1405-1416

6. Liu L, Johnson HL, Cousens S, Perin J, Scott S, Lawn JE, Rudan I, Campbell $\mathrm{H}$, Cibulskis R, Li M, Mathers C, Child Health Epidemiology Reference
Group of WHO and UNICEF (2012) Global, regional, and national causes of child mortality: an updated systematic analysis for 2010 with time trends since 2000. Lancet 379:2151-2161

7. Brock JH (1980) Lactoferrin in human milk: its role in iron absorption and protection against enteric infection in the newborn infant. Arch Dis Child $55: 417-421$

8. Corthesy B (2013) Role of secretory lgA in infection and maintenance of homeostasis. Autoimmun Rev 12:661-665

9. Davis KM, Weiser JN (2011) Modifications to the peptidoglycan backbone help bacteria to establish infection. Infect Immun 79:562-570

10. Cooper CA, Maga EA, Murray JD (2015) Production of human lactoferrin and lysozyme in the milk of transgenic dairy animals: past, present, and future. Transgenic Res 24:605-614

11. Vanderkelen L, Ons E, Van Herreweghe JM, Callewaert L, Goddeeris BM, Michiels CW (2012) Role of lysozyme inhibitors in the virulence of avian pathogenic Escherichia coli. PLoS One 7:e45954

12. Ellison RT, Giehl TJ (1991) Killing of gram-negative bacteria by lactoferrin and lysozyme. J Clin Invest 88:1080-1091

13. Masschalck B, Michiels CW (2003) Antimicrobial properties of lysozyme in relation to foodborne vegetative bacteria. Crit Rev Microbiol 29:191-214

14. Donaldson DM, Roberts RR, Larsen HS, Tew JG (1974) Interrelationship between serum beta-lysin, lysozyme, and the antibody-complement system in killing Escherichia coli. Infect Immun 10:657-666

15. Torsteinsdottir I, Hakansson L, Hallgren R, Gudbjornsson B, Arvidson NG, Venge $P$ (1999) Serum lysozyme: a potential marker of monocyte/macrophage activity in rheumatoid arthritis. Rheumatology 38:1249-1254

16. Chandan RC, Parry RM, Shahani KM (1968) Lysozyme lipase and ribonuclease in milk of various species. J Dairy Sci 51:606-607

17. Tong J, Wei H, Liu X, Hu W, Bi M, Wang Y, Li Q, Li N (2011) Production of recombinant human lysozyme in the milk of transgenic pigs. Transgenic Res 20:417-419

18. Cooper CA, Klobas LCG, Maga EA, Murray JD (2013) Consuming transgenic goats' milk containing the antimicrobial protein lysozyme helps resolve diarrhea in young pigs. PLoS One 8:e58409

19. Maga EA, Desai PT, Weimer BC, Nguyet D, Kueltz D, Murray JD (2012) Consumption of lysozyme-rich milk can alter microbial fecal populations. Appl Environ Microb 78:6153-6160

20. Lu D, Liu S, Ding F, Wang H, Li J (2016) Large-scale production of functional human lysozyme from marker-free transgenic cloned cows. Sci Rep 6:22947

21. Lu D, Li Q, Wu Z, Shang S, Liu S, Wen X, Li Z, Wu F, Li N (2014) High-level recombinant human lysozyme expressed in milk of transgenic pigs can inhibit the growth of Escherichia coli in the duodenum and influence intestinal morphology of sucking pigs. PLoS One 9:e89130

22. Lu D, Liu S, Shang S, Wu F, Wen X, Li Z, Li Y, Hu X, Zhao Y, Li Q, Li N (2015) Production of transgenic-cloned pigs expressing large quantities of recombinant human lysozyme in milk. PLoS One 10:e0123551

23. Zhou D, Zhu YH, Zhang W, Wang ML, Fan WY, Song D, Yang GY, Jensen BB, Wang JF (2015) Oral administration of a select mixture of Bacillus probiotics generates Tr1 cells in weaned F4ab/acR- pigs challenged with an F4+ ETECNTEC/EPEC strain. Vet Res 46:95

24. Hur J, Lee JH (2012) Comparative evaluation of the vaccine candidate expressing against enterotoxigenic Escherichia coli (ETEC) adhesions is colibacillosis with a commercial vaccine using a pig model. Vaccine 30:3829-3833

25. Chandler DS, Mynott TL (1998) Bromelain protects piglets from diarrhoea caused by oral challenge with K88 positive enterotoxigenic Escherichia coli. Gut 43:196-202

26. Vu-Khac H, Holoda E, Pilipcinec E, Blanco M, Blanco JE, Dahbi G, Mora A Lopez C, Gonzalez EA, Blanco J (2007) Serotypes, virulence genes, intimin types and PFGE profiles of Escherichia coli isolated from piglets with diarrhoea in Slovakia. Vet J 174:176-187

27. Peterson LW, Artis D (2014) Intestinal epithelial cells: regulators of barrier function and immune homeostasis. Nat Rev Immunol 14:141-153

28. Skern R, Frost P, Nilsen F (2005) Relative transcript quantification by quantitative PCR: roughly right or precisely wrong? BMC Mol Biol 6:10

29. Ji S, Zhang H, Yan H, Azarfar A, Shi H, Alugongo G, Li S, Cao Z, Wang Y (2017) Comparison of rumen bacteria distribution in original rumen digesta, rumen liquid and solid fractions in lactating Holstein cows. J Anim Sci Biotechnol 8:16 
30. Heijs SK, Haese RR, van der Wielen PW, Forney LJ, van Elsas JD (2007) Use of $16 \mathrm{~S}$ rRNA gene based clone libraries to assess microbial communities potentially involved in anaerobic methane oxidation in a Mediterranean cold seep. Microb Ecol 53:384-398

31. Dewhirst FE, Chen T, Izard J, Paster BJ, Tanner AC, Yu WH, Lakshmanan A, Wade WG (2010) The human oral microbiome. J Bacteriol 192:5002-5017

32. Ibrahim HR, Imazato K, Ono H (2011) Human lysozyme possesses novel antimicrobial peptides within its $\mathrm{N}$-terminal domain that target bacterial respiration. J Agric Food Chem 59:10336-10345

33. Ibrahim HR, Aoki T, Pellegrini A (2002) Strategies for new antimicrobial proteins and peptides: lysozyme and aprotinin as model molecules. Curr Pharm Des 8:671-693

34. Ragland SA, Criss AK (2017) From bacterial killing to immune modulation: recent insights into the functions of lysozyme. PLoS Pathog 13:e1006512

35. Kau AL, Ahern PP, Griffin NW, Goodman AL, Gordon JI (2011) Human nutrition, the gut microbiome and the immune system. Nature 474:327-336

36. Yang KM, Jiang ZY, Zheng CT, Wang L, Yang XF (2014) Effect of Lactobacillus plantarum on diarrhea and intestinal barrier function of young piglets challenged with enterotoxigenic Escherichia coli K88. J Anim Sci 92:1496-1503

37. Melkebeek V, Goddeeris BM, Cox E (2013) ETEC vaccination in pigs. Vet Immunol Immunopathol 152:37-42

38. Hoijer MA, Melief MJ, Debets R, Hazenberg MP (1997) Inflammatory properties of peptidoglycan are decreased after degradation by human N-acetylmuramyl-L-alanine amidase. Eur Cytokine Netw 8:375-381

39. Lee W, Ku S, Na DH, Bae J (2015) Anti-inflammatory effects of lysozyme against HMGB1 in human endothelial cells and in mice. Inflammation 38:1911-1924
40. Alexa P, Hamrik J, Konstantinova L, Sramkova-Zajacova Z (2011) Experimental infection of weaned piglets with enterotoxigenic Escherichia coli O149:F4. Acta Vet Brno 80:337-341

41. Virdi V, Coddens A, De Buck S, Millet S, Goddeeris BM, Cox E, De Greve H, Depicker A (2013) Orally fed seeds producing designer IgAs protect weaned piglets against enterotoxigenic Escherichia coli infection. Proc Natl Acad Sci U S A 110:11809-11814

42. Landers TF, Cohen B, Wittum TE, Larson EL (2012) A review of antibiotic use in food animals: perspective, policy, and potential. Public Health Rep 127:4-22

43. Oliver WT, Wells JE (2015) Lysozyme as an alternative to growth promoting antibiotics in swine production. J Anim Sci Biotechnol 6:35

44. Garas LC, Cooper CA, Dawson MW, Wang JL, Murray JD, Maga EA (2017) Young pigs consuming lysozyme transgenic goat milk are protected from clinical symptoms of enterotoxigenic Escherichia coli infection. J Nutr 147:2050-2059

45. Cooper CA, Maga EA, Murray JD (2014) Consumption of transgenic milk containing the antimicrobials lactoferrin and lysozyme separately and in conjunction by 6-week-old pigs improves intestinal and systemic health. J Dairy Res 81:30-37

46. Wolf E, Schernthaner W, Zakhartchenko V, Prelle K, Stojkovic M, Brem G (2000) Transgenic technology in farm animals - progress and perspectives. Exp Physiol 85:615-625

47. Niemann H, Kues W, Carnwath JW (2009) Transgenic farm animals: current status and perspectives for agriculture and biomedicine. In: Engelhard M, Hagen K, Boysen M (eds) Genetic engineering in livestock Ethics of science and technology assessment, vol 34. Springer, Berlin

\section{Submit your next manuscript to BioMed Central and we will help you at every step:}

- We accept pre-submission inquiries

- Our selector tool helps you to find the most relevant journal

- We provide round the clock customer support

- Convenient online submission

- Thorough peer review

- Inclusion in PubMed and all major indexing services

- Maximum visibility for your research

Submit your manuscript at www.biomedcentral com/submit
O Biomed Central 